\title{
Existence Results for a Coupled System of Nonlinear Fractional Boundary Value Problems at Resonance
}

\author{
Ruijuan Liu, ${ }^{1,2}$ Chunhai Kou, ${ }^{3}$ and Xiujuan Xie ${ }^{3}$ \\ ${ }^{1}$ College of Information Science and Technology, Donghua University, Shanghai 201620, China \\ ${ }^{2}$ College of Fundamental Studies, Shanghai University of Engineering Science, Shanghai 201620, China \\ ${ }^{3}$ Department of Applied Mathematics, Donghua University, Shanghai 201620, China
}

Correspondence should be addressed to Ruijuan Liu; ruirui0516@163.com

Received 30 April 2013; Accepted 15 July 2013

Academic Editor: Yong-Kui Chang

Copyright (c) 2013 Ruijuan Liu et al. This is an open access article distributed under the Creative Commons Attribution License, which permits unrestricted use, distribution, and reproduction in any medium, provided the original work is properly cited.

\begin{abstract}
Some new Banach spaces are established. Based on those new Banach spaces and by using the coincidence degree theory, we present the existence results for a coupled system of nonlinear fractional differential equations with multipoint boundary value conditions at resonance case.
\end{abstract}

\section{Introduction}

Fractional differential equations (FDEs) have been of great interest for the last three decades. It is caused both by the intensive development of the theory of fractional calculus itself and by the applications of such constructions in the modeling of many phenomena in various fields of science and engineering. Indeed, we can find numerous applications in viscoelasticity, electrochemistry, control, porous media, and so forth. Therefore, the theory of FDEs has been developed very quickly. Many qualitative theories of FDEs have been obtained. Many important results can be found in [1-13] and the references cited therein.

However, there are few articles which consider the boundary value problems at resonance for a coupled system of nonlinear fractional differential equations. In [14], the authors investigated the existence and uniqueness of solutions for the multipoint boundary value problems for fractional differential equations of the form

$$
\begin{aligned}
& D_{t}^{\alpha} y(t)=f\left(t, y(t), D_{t}^{\beta} y(t)\right), \quad t \in(0,1), \\
& y(0)=0, \quad D_{t}^{\beta} y(1)-\sum_{i=1}^{m-2} \varsigma_{i} D_{t}^{\beta} y\left(\xi_{i}\right)=y_{0},
\end{aligned}
$$

where $1<\alpha \leq 2,0<\beta<1,0<\xi_{i}<1(i=1,2, \ldots, m-$ 2), $\varsigma_{i} \geq 0$, with $\gamma=\sum_{i=1}^{m-2} \varsigma_{i} \xi_{i}^{\alpha-\beta-1}<1$ and $D_{t}^{\alpha}$ represents the standard Riemann-Liouville fractional derivative. And the analysis relies on the Schauder fixed point theorem and the Banach contraction principle.

In [15], the following coupled system of nonlinear fractional differential equation boundary value problem:

$$
\begin{gathered}
D_{0+}^{\alpha} u(t)=f\left(t, v(t), D_{0+}^{\beta-1} v(t)\right), \quad 0<t<1, \\
D_{0+}^{\beta} v(t)=g\left(t, u(t), D_{0+}^{\alpha-1} u(t)\right), \quad 0<t<1, \\
u(0)=v(0)=0, \quad u(1)=\sigma_{1} u\left(\eta_{1}\right), \\
v(1)=\sigma_{2} v\left(\eta_{2}\right)
\end{gathered}
$$

was considered. Where $1<\alpha, \beta \leq 2,0<\eta_{1}, \eta_{2}<1, \sigma_{1}$, $\sigma_{2}>0, \sigma_{1} \eta_{1}{ }^{\alpha-1}=\sigma_{2} \eta_{2}{ }^{\beta-1}, D$ is the standard RiemannLiouville fractional derivative and $f, g:[0,1] \times R^{2} \rightarrow R$ are continuous. For more works about coupled system involving fractional differential equations, for instance, see $[16,17]$.

In this paper, we investigate the existence of solutions for the boundary value problem with the following form:

$$
\begin{array}{ll}
D_{0+}^{\alpha} u(t)=f\left(t, v(t), D_{0+}^{p} u(t)\right), & t \in(0,1), \\
D_{0+}^{\beta} v(t)=g\left(t, u(t), D_{0+}^{q} v(t)\right), & t \in(0,1),
\end{array}
$$




$$
\begin{array}{ll}
u(0)=0, & D_{0+}^{p} u(1)=\sum_{i=1}^{m-2} a_{i} D_{0+}^{p} u\left(\xi_{i}\right), \\
v(0)=0, & D_{0+}^{q} v(1)=\sum_{i=1}^{m-2} b_{i} D_{0+}^{q} v\left(\eta_{i}\right),
\end{array}
$$

where $1<\alpha, \beta \leq 2,0<p, q<1, \alpha-p-1, \beta-q-1 \geq 0, a_{i}$, $b_{i} \geq 0,0<\xi_{i}$, and $\eta_{i}<1(i=1,2, \ldots, m-2)$. Moreover, at resonance case,

$$
\sum_{i=1}^{m-2} a_{i} \xi_{i}^{\alpha-p-1}=1, \quad \sum_{i=1}^{m-2} b_{i} \eta_{i}^{\beta-q-1}=1,
$$

a sufficient condition for the existence of solutions is established by the coincidence degree continuation theorem.

\section{Background Materials and Preliminaries}

For the convenience of the reader, we present here some necessary basic knowledge and definitions about fractional calculus theory. These definitions and lemmas can be found in $[4-6]$.

Definition 1. The fractional integral of order $\alpha>0$ of a function $f:(0, \infty) \rightarrow R$ is given by

$$
I_{0+}^{\alpha} f(t)=\frac{1}{\Gamma(\alpha)} \int_{0}^{t}(t-s)^{\alpha-1} f(s) d s,
$$

provided the right side is pointwise defined on $(0, \infty)$.

Definition 2. The fractional derivative of order $\alpha>0$ of a function $f:(0, \infty) \rightarrow R$ is given by

$$
D_{0+}^{\alpha} f(t)=\frac{1}{\Gamma(n-\alpha)}\left(\frac{d}{d t}\right)^{n} \int_{0}^{t}(t-s)^{n-\alpha-1} f(s) d s,
$$

where $n=[\alpha]+1$, provided the right side is pointwise defined on $(0, \infty)$.

It can be directly verified that the Riemann-Liouville fractional integration and fractional differentiation operators of the power functions $t^{\beta}$ yield power functions of the same form. For $\alpha \geq 0, \beta>-1$, there are

$$
I_{0+}^{\alpha} t^{\beta}=\frac{\Gamma(\beta+1)}{\Gamma(\beta+\alpha+1)} t^{\beta+\alpha}, \quad D_{0+}^{\alpha} t^{\beta}=\frac{\Gamma(\beta+1)}{\Gamma(\beta-\alpha+1)} t^{\beta-\alpha} .
$$

Lemma 3. Assume that $\alpha>0, y \in C(0,1) \cap L^{1}[0,1]$, then the differential equation $D_{0+}^{\alpha} y(t)=0$ has solutions

$$
y(t)=c_{1} t^{\alpha-1}+c_{2} t^{\alpha-2}+\cdots+c_{n} t^{\alpha-n},
$$

where $c_{i} \in R, i=1,2, \ldots, n, n=[\alpha]+1$.

Lemma 4. Assume that $y \in C(0,1) \cap L^{1}[0,1]$ and $D_{0+}^{\alpha} y \in$ $C(0,1) \cap L^{1}[0,1]$, then $I_{0+}^{\alpha} D_{0+}^{\alpha} y(t)=y(t)+c_{1} t^{\alpha-1}+c_{2} t^{\alpha-2}+$ $\cdots+c_{n} t^{\alpha-n}$, where $c_{i} \in R, i=1,2, \ldots, n, n=[\alpha]+1$.
Lemma 5. Let $\alpha>\beta>0, t \in[0,1]$ and $y(t) \in L^{1}[0,1]$, and then

$$
\begin{aligned}
& I_{0+}^{\alpha} I_{0+}^{\beta} y(t)=I_{0+}^{\alpha+\beta} y(t), \quad D_{0+}^{\alpha} I_{0+}^{\alpha} y(t)=y(t), \\
& D_{0+}^{\beta} I_{0+}^{\alpha} y(t)=I_{0+}^{\alpha-\beta} y(t) .
\end{aligned}
$$

The following definitions and coincidence degree theory are fundamental in the proofs of our main results.

Definition 6. Let $Y$ and $Z$ be Banach spaces. A linear mapping $L: \operatorname{dom}(L) \subset Y \rightarrow Z$ is called a Fredholm mapping if the following two conditions hold:

(1) Ker $L$ has a finite dimension;

(2) $\operatorname{Im} L$ is closed and has a finite codimension.

If $L$ is a Fredholm mapping, its (Fredholm) index is the integer ind $L=\operatorname{dim} \operatorname{Ker} L-\operatorname{codim} \operatorname{Im} L$.

And if $L$ is a Fredholm mapping of index zero, there exist linear continuous projectors $P: Y \rightarrow Y$ and $Q: Z \rightarrow Z$ such that $\operatorname{Im} P=\operatorname{Ker} L, \operatorname{Ker} Q=\operatorname{Im} L$, and $Y=\operatorname{Ker} L \oplus$ $\operatorname{Ker} P, Z=\operatorname{Im} L \oplus \operatorname{Im} Q$. Then it follows that $\left.L\right|_{\operatorname{dom} L \cap K e r} P$ : $\operatorname{dom} L \cap \operatorname{Ker} P \rightarrow \operatorname{Im} L$ is invertible. We denote the inverse of this map by $K_{P}: \operatorname{Im} L \rightarrow \operatorname{dom} L \cap \operatorname{Ker} P$. The generalized inverse of this map is denoted by $K_{P, Q}: Y \rightarrow \operatorname{dom} L \cap \operatorname{Ker} P$, $K_{P, Q}=K_{P}(I-Q)$.

Definition 7. Assume that $L: \operatorname{dom} L \subset Y \rightarrow Z$ is a Fredholm mapping, and if $\Omega$ is an open bounded subset of $Y$, the map $N$ will be called $L$-compact on $\bar{\Omega}$ if $Q N: \Omega \rightarrow Z$ is bounded and $K_{P, Q} N: \Omega \rightarrow Y$ is compact.

If $\operatorname{Im} Q$ is isomorphic to Ker $L$, there exists an isomorphism $J_{N L}: \operatorname{Im} Q \rightarrow \operatorname{Ker} L$. We have the coincidence degree continuation theorem which is proved in [18].

Theorem 8. Assume that $L$ is a Fredholm mapping of index zero and let $N$ be L-compact on $\bar{\Omega}$, where $\Omega$ is an open bounded subset of $Y$. Suppose that the following conditions are satisfied:

(1) $L y \neq \lambda N y$ for each $(y, \lambda) \in[(\operatorname{dom} L \backslash \operatorname{Ker} L) \cap \partial Q] \times$ $(0,1)$;

(2) $N y \notin \operatorname{Im} L$ for each $y \in \operatorname{Ker} L \cap \partial Q$;

(3) $\operatorname{deg}\left(\left.J_{N L} Q N\right|_{\operatorname{Ker} L}, \Omega \cap \operatorname{Ker} L, 0\right) \neq 0$, where $Q: Z \rightarrow$ $Z$ is a continuous projection as mentioned above with $\operatorname{Im} L=\operatorname{Ker} Q$ and $J_{N L}: \operatorname{Im} Q \rightarrow \operatorname{Ker} L$ is any isomorphism.

Then the equation $L y=N y$ has at least one solution in $\operatorname{dom} L \cap$ $\bar{\Omega}$.

Let $Z_{1}=L^{1}[0,1]$ with the norm $\|x\|_{1}=\int_{0}^{1}|x(s)| d s$. For $0<p, q<1$, we define a linear space

$$
\begin{aligned}
& C^{p}[0,1], C^{q}[0,1]: \\
& \begin{aligned}
Y_{1} & =C^{p}[0,1] \\
& =\left\{u(t) \mid u(t)=I_{0+}^{p} x(t), x \in C[0,1], t \in[0,1]\right\},
\end{aligned}
\end{aligned}
$$




$$
\begin{aligned}
Y_{2} & =C^{q}[0,1] \\
& =\left\{v(t) \mid v(t)=I_{0+}^{q} y(t), y \in C[0,1], t \in[0,1]\right\},
\end{aligned}
$$

to be accompanied by

$$
\begin{aligned}
& \|u\|_{Y_{1}}=\max _{t \in[0,1]}\left|D_{0+}^{p} u(t)\right|+\max _{t \in[0,1]}|u(t)|, \\
& \|v\|_{Y_{2}}=\max _{t \in[0,1]}\left|D_{0+}^{q} v(t)\right|+\max _{t \in[0,1]}|v(t)| .
\end{aligned}
$$

We can prove that $Y=Y_{1} \times Y_{2}$ is a Banach space with the norm $\|(u, v)\|_{Y}=\max \left\{\|u\|_{Y_{1}},\|u\|_{Y_{2}}\right\}$, and $Z=Z_{1} \times Z_{1}$ is a Banach space, with the $\operatorname{norm}\|(x, y)\|_{Z}=\max \left\{\|x\|_{1},\|y\|_{1}\right\}$.

Let $L_{1}$ to be the linear operator from $\operatorname{dom} L_{1} \cap Y_{1}$ to $Z_{1}$ with

$$
\begin{aligned}
\operatorname{dom} L_{1}=\left\{u \in C^{p}[0,1] \mid D_{0+}^{\alpha} u \in L^{1}[0,1],\right. \\
\left.u(0)=0, D_{0+}^{p} y(1)=\sum_{i=1}^{m-2} a_{i} D_{0+}^{p} u\left(\xi_{i}\right)\right\} .
\end{aligned}
$$

And $L_{1} u=D_{0+}^{\alpha} u, u \in \operatorname{dom} L_{1}$. Let $L_{2}$ be the linear operator from dom $L_{2} \cap Y_{2}$ to $Z_{1}$ with

$$
\begin{aligned}
\operatorname{dom} L_{2}=\left\{v \in C^{q}[0,1] \mid D_{0+}^{\beta} v \in L^{1}[0,1],\right. \\
\left.v(0)=0, D_{0+}^{q} v(1)=\sum_{j=1}^{m-2} b_{j} D_{0+}^{q} v\left(\eta_{j}\right)\right\} .
\end{aligned}
$$

And $L_{1} v=D_{0+}^{\beta} v, v \in \operatorname{dom} L_{2}$. Define $L$ to be the linear operator from dom $L \cap Y$ to $Z$ with

$$
\operatorname{dom} L=\left\{(u, v) \in Y \mid u \in \operatorname{dom} L_{1}, v \in \operatorname{dom} L_{2}\right\},
$$

and $L(u, v)=\left(L_{1} u, L_{2} v\right)$. We define $N: Y \rightarrow Z$ by setting

$$
N(u, v)=\left(N_{1}(u(t), v(t)), N_{2}(u(t), v(t))\right),
$$

where $N_{1}(u(t), v(t))=f\left(t, v(t), D_{0+}^{p} u(t)\right), \quad N_{2}(u(t), v(t))=$ $g\left(t, u(t), D_{0+}^{q} v(t)\right)$.

Then the coupled system of BVPs (3) can be written as $L(u, v)=N(u, v)$.

Lemma 9 (see [19]). $F \subset C^{\mu}[0,1], 0<\mu<1$, is a sequentially compact set if and only if $F$ is uniformly bounded and equicontinuous. Here uniformly bounded means that there exists $M>0$ such that for every $u \in F$,

$$
\|y\|_{C^{\mu}}=\max _{t \in J}\left|D_{0+}^{\mu} u(t)\right|+\max _{t \in J}|u(t)|<M,
$$

and equicontinuous means that for all $\varepsilon>0, \exists \delta>0$, for all $t_{1}, t_{2} \in[0,1],\left|t_{1}-t_{2}\right|<\delta, u \in F$, there hold $\left|u\left(t_{1}\right)-u\left(t_{2}\right)\right|<$ $\varepsilon,\left|D_{0+}^{\beta} u\left(t_{1}\right)-D_{0+}^{\beta} u\left(t_{2}\right)\right|<\varepsilon$.
Definition 10 (see [19]). One says that $f:[0,1] \times R^{2} \rightarrow R$ satisfies the Carathéodory condition if (i) for each $(x, y) \in$ $R^{2}$, the function $t \in[0,1] \rightarrow f(t, x, y) \in R$ is measurable on $[0,1]$; (ii) for a.e. $t \in[0,1]$, the function $(x, y) \in R^{2} \rightarrow$ $f(t, x, y) \in R$ is continuous on $R^{2}$; (iii) for each $r>0$, there exists $\varphi_{r}(t) \in L^{1}[0,1]$ such that $|f(t, x, y)| \leq \varphi_{r}(t)$ for a.e. $t \in[0,1]$ and all $(x, y) \in R^{2}$ with $\sqrt{x^{2}+y^{2}} \leq r$.

\section{Main Results}

In this section, we will give and prove our main results.

Lemma 11. Let $f, g \in Z_{1}, J=[0,1], \sum_{i=1}^{m-2} a_{i} \xi_{i}^{\alpha-p-1}=1$, $\sum_{i=1}^{m-2} b_{i} \eta_{i}^{\beta-q-1}=1$. Then $u, v \in Y$ are, respectively, the solutions of the following boundary value problems:

$$
\begin{gathered}
D_{0+}^{\alpha} u(t)=f(t), \quad t \in J, \\
u(0)=0, \quad D_{0+}^{p} u(1)=\sum_{i=1}^{m-2} a_{i} D_{0+}^{p} u\left(\xi_{i}\right), \\
D_{0+}^{\beta} v(t)=g(t), \quad t \in J, \\
v(0)=0, \quad D_{0+}^{q} v(1)=\sum_{i=1}^{m-2} b_{i} D_{0+}^{q} v\left(\eta_{i}\right)
\end{gathered}
$$

if and only if $u, v$ satisfied

$$
\begin{gathered}
u(t)=c_{1} t^{\alpha-1}+I_{0+}^{\alpha} f(t), \\
\sum_{i=1}^{m-2} a_{i} \xi_{i}^{\alpha-p-1} \int_{0}^{1}(1-s)^{\alpha-p-1} f(s) d s \\
=\int_{0}^{\xi_{i}}\left(\xi_{i}-s\right)^{\alpha-p-1} f(s) d s, \\
v(t)=c_{2} t^{\beta-1}+I_{0+}^{\beta} g(t), \\
\sum_{i=1}^{m-2} b_{i} \eta_{i}^{\beta-q-1} \int_{0}^{1}(1-s)^{\beta-q-1} g(s) d s \\
=\int_{0}^{\eta_{i}}\left(\eta_{i}-s\right)^{\beta-q-1} g(s) d s .
\end{gathered}
$$

Proof. $(u, v)$ in $Y_{1} \times Y_{2}$ are the solutions of the following boundary value problem:

$$
\begin{aligned}
& I_{0+}^{\alpha} D_{0+}^{\alpha} u(t)=I_{0+}^{\alpha} f(t)=\frac{1}{\Gamma(\alpha)} \int_{0}^{t}(t-s)^{\alpha-1} f(s) d s, \\
& I_{0+}^{\beta} D_{0+}^{\beta} u(t)=I_{0+}^{\beta} g(t)=\frac{1}{\Gamma(\beta)} \int_{0}^{t}(t-s)^{\beta-1} g(s) d s .
\end{aligned}
$$

According to Lemma 4, we have

$$
\begin{aligned}
& u(t)=c_{1} t^{\alpha-1}+c_{2} t^{\alpha-2}+I_{0+}^{\alpha} f(t), \\
& v(t)=c_{3} t^{\beta-1}+c_{4} t^{\beta-2}+I_{0+}^{\beta} g(t) .
\end{aligned}
$$


$\operatorname{By} u(0)=v(0)=0$, we get $c_{2}=c_{4}=0$, and then $u(t)=c_{1} t^{\alpha-1}+$ $I_{0+}^{\alpha} f(t), v(t)=c_{3} t^{\beta-1}+I_{0+}^{\beta} g(t)$.

Using (7) and Lemma 5, we get that

$$
\begin{aligned}
D_{0+}^{p} u(t) & =D_{0+}^{p} I_{0+}^{\alpha} f(t)+\frac{c_{1} \Gamma(\alpha)}{\Gamma(\alpha-p)} t^{\alpha-p-1} \\
& =I_{0+}^{\alpha-p} f(t)+\frac{c_{1} \Gamma(\alpha)}{\Gamma(\alpha-p)} t^{\alpha-p-1}, \\
D_{0+}^{q} v(t) & =D_{0+}^{q} I_{0+}^{\beta} g(t)+\frac{c_{3} \Gamma(\beta)}{\Gamma(\beta-q)} t^{\beta-q-1} \\
& =I_{0+}^{\beta-q} g(t)+\frac{c_{3} \Gamma(\alpha)}{\Gamma(\beta-q)} t^{\beta-q-1} .
\end{aligned}
$$

By the boundary conditions, $\sum_{i=1}^{m-2} a_{i} \xi_{i}^{\alpha-p-1}=1, \sum_{i=1}^{m-2}$ $b_{i} \eta_{i}^{\beta-q-1}=1$, we have

$$
\begin{gathered}
\sum_{i=1}^{m-2} a_{i} \xi_{i}^{\alpha-p-1} \int_{0}^{1}(1-s)^{\alpha-p-1} f(s) d s \\
=\int_{0}^{\xi_{i}}\left(\xi_{i}-s\right)^{\alpha-p-1} f(s) d s, \\
\sum_{i=1}^{m-2} b_{i} \eta_{i}^{\beta-q-1} \int_{0}^{1}(1-s)^{\beta-q-1} g(s) d s \\
=\int_{0}^{\eta_{i}}\left(\eta_{i}-s\right)^{\beta-q-1} g(s) d s .
\end{gathered}
$$

Conversely, suppose that (23) and (24) hold. In view of Lemma 5, we can easily verify that $u, v$ are the solutions of (17) and (18), respectively. Then, we complete the proof.

Lemma 12. The mapping $L: \operatorname{dom}(L) \subset Y \rightarrow Z$ is a Fredholm operator of index zero.

Proof. Since Ker $L_{1}=\left\{u \mid D_{0+}^{\alpha} u(t)=0\right\}, \operatorname{Ker} L_{2}=\{v \mid$ $\left.D_{0+}^{\beta} v(t)=0\right\}$, it can be directly verified that $\operatorname{Ker} L_{1}=\{u \mid$ $\left.u=c_{1} t^{\alpha-1}, c_{1} \in R\right\}, \operatorname{Ker} L_{2}=\left\{v \mid v=c_{2} t^{\beta-1}, c_{2} \in R\right\}$. It is clear that $\operatorname{Ker} L=\left\{c_{1} t^{\alpha-1}, c_{2} t^{\beta-1} \mid c_{1}, c_{2} \in R\right\}$.

Let $(x, y) \in \operatorname{Im} L$; then there exists $(u, v) \in \operatorname{dom} L$ such that $(x, y)=L(u, v)$, that is, $u \in Y_{1}, x=D_{0+}^{\alpha} u$ and $v \in Y_{2}$, $y=D_{0+}^{\beta} v$. From these results, we can easily deduce the results

$$
\operatorname{Im} L=\{(x, y) \in Z \mid x \text { satisfies (23), } y \text { satisfies (24) }\} \text {. }
$$

Let $t \in(0,1), \sum_{i=0}^{m-2} a_{i} \xi_{i}^{\alpha-p-1}=1, \sum_{i=0}^{m-2} b_{i} \eta_{i}^{\beta-q-1}=1$. Assume that

$$
\begin{array}{r}
\Lambda_{1}=\sum_{i=1}^{m-2} a_{i}\left(\xi_{i}^{\alpha-p-1} \int_{0}^{1}(1-s)^{\alpha-p-1} s^{k} d s\right. \\
\left.-\int_{0}^{\xi_{i}}\left(\xi_{i}-s\right)^{\alpha-p-1} s^{k} d s\right) \neq 0,
\end{array}
$$

$$
\begin{aligned}
\Lambda_{2}= & \sum_{i=1}^{m-2} b_{i}\left(\eta_{i}^{\beta-q-1} \int_{0}^{1}(1-s)^{\beta-q-1} s^{k} d s\right. \\
& \left.-\int_{0}^{\eta_{i}}\left(\eta_{i}-s\right)^{\beta-q-1} s^{k} d s\right) \neq 0 .
\end{aligned}
$$

Consider the auxiliary mapping,

$$
\begin{array}{r}
Q_{1}: Z \longrightarrow R: Q_{1} x=\sum_{i=1}^{m-2} a_{i}\left(\xi_{i}^{\alpha-p-1} \int_{0}^{1}(1-s)^{\alpha-p-1} x(s) d s\right. \\
\left.-\int_{0}^{\xi_{i}}\left(\xi_{i}-s\right)^{\alpha-p-1} x(s) d s\right), \\
Q_{2}: Z \longrightarrow R: Q_{2} y=\sum_{i=1}^{m-2} b_{i}\left(\eta_{i}^{\beta-q-1} \int_{0}^{1}(1-s)^{\beta-q-1} y(s) d s\right. \\
\left.-\int_{0}^{\eta_{i}}\left(\eta_{i}-s\right)^{\beta-q-1} y(s) d s\right) .
\end{array}
$$

It is obvious that $Q_{1}, Q_{2}$ are continuous linear mappings. Also we have that $Q_{1}\left(t^{k}\right)=\Lambda_{1}, Q_{2}\left(t^{k}\right)=\Lambda_{2}$. Take the mapping $Q: Z \rightarrow Z$ defined by $Q(x, y)=\left(Q_{1}^{\prime} x, Q_{2}^{\prime} y\right)$. $Q_{1}^{\prime} x=\left(Q_{1} x / \Lambda_{1}\right) t^{k}, Q_{2}^{\prime} x=\left(Q_{2} x / \Lambda_{2}\right) t^{k}$, where $x, y \in Z$. Note that $(x, y) \in \operatorname{Im} L$ implies $Q(x, y)=\left(Q_{1} x, Q_{2} y\right)=(0,0)$. Evidently,

$$
\operatorname{Im} Q=\left\{(x, y) \mid x=c_{1} t^{k}, y=c_{2} t^{k}, c_{1}, c_{2} \in R\right\},
$$

and $Q: Z \rightarrow Z$ is a continuous linear projector. In fact, for any $(x, y) \in Z$, we have

$$
\begin{gathered}
Q_{1}\left(Q_{1}^{\prime} x\right)=Q_{1}\left(\frac{Q_{1} x}{\Lambda_{1}} t^{k}\right)=\frac{Q_{1} x}{\Lambda_{1}} Q_{1}\left(t^{k}\right)=\frac{Q_{1} x}{\Lambda_{1}} \Lambda_{1}=Q_{1} x \\
Q_{2}\left(Q_{2}^{\prime} y\right)=Q_{2}\left(\frac{Q_{2} y}{\Lambda_{2}} t^{k}\right)=\frac{Q_{2} y}{\Lambda_{2}} Q_{2}\left(t^{k}\right)=\frac{Q_{2} y}{\Lambda_{2}} \Lambda_{2}=Q_{2} y \\
Q_{1}^{\prime 2} x=Q_{1}^{\prime}\left(Q_{1}^{\prime} x\right)=\frac{Q_{1}\left(Q_{1}^{\prime} x\right)}{\Lambda_{1}} t^{k}=\frac{Q_{1} x}{\Lambda_{1}} t^{k}=Q_{1}^{\prime} x \\
Q_{2}^{\prime 2} y=Q_{2}^{\prime}\left(Q_{2}^{\prime} y\right)=\frac{Q_{2}\left(Q_{2}^{\prime} y\right)}{\Lambda_{2}} t^{k}=\frac{Q_{2} y}{\Lambda_{2}} t^{k}=Q_{2}^{\prime} y
\end{gathered}
$$

that is to say, $Q: Z \rightarrow Z$ is idempotent.

Observe that $(x, y) \in \operatorname{Im} L$ leads to $Q(x, y)=(0,0)$ and $(x, y) \in \operatorname{Ker} Q$, where $(0,0)$ is the element in $Z$. Conversely, if $(x, y) \in \operatorname{Ker} Q$, we can have that $Q(x, y)=(0,0)$, that is to say, $(x, y) \in \operatorname{Im} L$. So, $\operatorname{Ker} Q=\operatorname{Im} L$.

Let $(x, y)=(x, y)-Q(x, y)+Q(x, y)=(I-Q)(x, y)+$ $Q(x, y)$, where $(x, y) \in Z$ is an arbitrary element. Since $Q(x, y) \in \operatorname{Im} Q$ and $(I-Q)(x, y) \in \operatorname{Ker} Q$, we obtain that $Z=\operatorname{Im} Q+\operatorname{Ker} Q$. Take $\left(x_{0}, y_{0}\right) \in \operatorname{Im} Q \cap \operatorname{Ker} Q,\left(x_{0}, y_{0}\right)$ can be written as $\left(x_{0}, y_{0}\right)=\left(c_{1} t^{k}, c_{2} t^{k}\right), c_{1}, c_{2} \in R$. For 
$\left(x_{0}, y_{0}\right) \in \operatorname{Im} Q$ then since $\left(x_{0}, y_{0}\right) \in \operatorname{Ker} Q=\operatorname{Im} L$, by $(27)$, we get that

$$
\begin{aligned}
Q\left(x_{0}, y_{0}\right) & =Q\left(c_{1} t^{k}, c_{2} t^{k}\right)=\left(c_{1} Q_{1}\left(t^{k}\right), c_{2} Q_{2}\left(t^{k}\right)\right) \\
& =\left(c_{1} \Lambda_{1}, c_{2} \Lambda_{2}\right)=(0,0),
\end{aligned}
$$

which implies that $\left(x_{0}, y_{0}\right)=(0,0)$. Therefore, $\operatorname{Im} Q \cap \operatorname{Ker} Q=$ $\{0,0\}$, and thus $Z=\operatorname{Im} Q \oplus \operatorname{Ker} Q=\operatorname{Im} Q \oplus \operatorname{Im} L$.

Now, $\operatorname{dim} \operatorname{Ker} L=1=\operatorname{dim} \operatorname{Im} Q=\operatorname{codim} \operatorname{Ker} Q=$ co $\operatorname{dim} \operatorname{Im} L<+\infty$, and observing that $\operatorname{Im} L$ is closed in $Z$, so $L$ is a Fredholm mapping of index zero.

Let $P: Y \rightarrow Y$ be defined by

$$
\begin{aligned}
& \left(P_{1} u\right)(t)=\left.D_{0+}^{p} u(t)\right|_{t=0} t^{\alpha-1}, \quad t \in J, u \in Y_{1}, \\
& \left(P_{2} v\right)(t)=\left.D_{0+}^{q} v(t)\right|_{t=0} t^{\beta-1}, \quad t \in J, v \in Y_{2},
\end{aligned}
$$

and $P(u, v)=\left(P_{1} u, P_{2} v\right)$ it is clear that $P_{1}, P_{2}, P$ are linear continuous projectors and $\operatorname{Im} P=\operatorname{Ker} L=\left\{c_{1} t^{\alpha-1}, c_{2} t^{\beta-1} \mid\right.$ $\left.c_{1}, c_{2} \in R\right\}$. Also, proceeding as the proof of Lemma 12, we can show that $Y=\operatorname{Im} P \oplus \operatorname{Ker} P=\operatorname{Ker} L \oplus \operatorname{Ker} P$.

Lemma 13. Assume that $f, g:[0,1] \times R^{2} \rightarrow R$ satisfies the Carathéodory conditions

$$
\begin{gathered}
\sum_{i=1}^{m-2} a_{i} \xi_{i}^{\alpha-p-1}=1, \quad \sum_{i=1}^{m-2} b_{i} \eta_{i}^{\beta-q-1}=1, \quad \alpha-p-1 \geq 0, \\
\beta-q-1 \geq 0, \quad \Lambda_{1} \neq 0, \quad \Lambda_{2} \neq 0 .
\end{gathered}
$$

Then $K_{P}(I-Q) N: Y \rightarrow Y$ is completely continuous.

Here we omit reasoning process, and it is easy to prove.

Consider the mapping $K_{P}: \operatorname{Im} L \rightarrow \operatorname{dom} L \cap \operatorname{Ker} P$, $K_{P}(x, y)=\left(I_{0+}^{\alpha} x, I_{0+}^{\beta} y\right)$,

$$
\begin{aligned}
\left(K_{P} x\right)(t) & =I_{0+}^{\alpha} x(t) \\
& =\frac{1}{\Gamma(\alpha)} \int_{0}^{t}(t-s)^{\alpha-1} x(s) d s, \quad x \in \operatorname{Im} L_{1}, \\
\left(K_{P} y\right)(t) & =I_{0+}^{\beta} y(t) \\
& =\frac{1}{\Gamma(\beta)} \int_{0}^{t}(t-s)^{\beta-1} y(s) d s, \quad y \in \operatorname{Im} L_{2} .
\end{aligned}
$$
$(x, y)$.

For $(x, y) \in \operatorname{Im} L$, we have $\left(K_{P} L\right)(x, y)=K_{P}(L(x, y))=$

$\left(L K_{P}\right)(x, y)=L\left(K_{P}(x, y)\right)=(x, y)$, for all $(x, y) \in \operatorname{Im} L$. Thus, $K_{P}=\left(L_{P}\right)^{-1}$, where $L_{P}=\left.L\right|_{\operatorname{dom} L \cap K e r P}: \operatorname{dom} L \cap$
Ker $P \rightarrow \operatorname{Im} L$; hence, for each $(x, y) \in \operatorname{Im} L$ and $t \in J$, we have

$$
\begin{aligned}
& \left\|K_{P}(x, y)\right\|_{Y} \\
& =\left\|\left(I_{0+}^{\alpha} x, I_{0+}^{\beta} y\right)\right\|_{Y} \\
& =\max _{t \in J}\left\{\left\|I_{0+}^{\alpha} x\right\|_{Y_{1}},\left\|I_{0+}^{\beta} y\right\|_{Y_{2}}\right\} \\
& =\max _{t \in J}\left\{\left\|D_{0+}^{p} I_{0+}^{\alpha} x\right\|+\left\|I_{0+}^{\alpha} x\right\|,\right. \\
& \left.\left\|D_{0+}^{q} I_{0+}^{\beta} y\right\|+\left\|I_{0+}^{\beta} x\right\|\right\} \\
& =\max _{t \in J}\left\{\left|\frac{1}{\Gamma(\alpha-p)} \int_{0}^{t}(t-s)^{\alpha-p-1} x(s) d s\right|\right. \\
& +\left|\frac{1}{\Gamma(\alpha)} \int_{0}^{t}(t-s)^{\alpha-1} x(s) d s\right|, \\
& \left|\frac{1}{\Gamma(\beta-q)} \int_{0}^{t}(t-s)^{\beta-q-1} y(s) d s\right| \\
& \left.+\left|\frac{1}{\Gamma(\beta)} \int_{0}^{t}(t-s)^{\beta-1} y(s) d s\right|\right\} \\
& \leq \max _{t \in J}\left\{\left(\frac{1}{\Gamma(\alpha-p)}+\frac{1}{\Gamma(\alpha)}\right)\|x\|_{1}\right. \text {, } \\
& \left.\left(\frac{1}{\Gamma(\beta-q)}+\frac{1}{\Gamma(\beta)}\right)\|y\|_{1}\right\} \\
& =\max _{t \in J}\left\{\eta_{1}\|x\|_{1}, \eta_{2}\|y\|_{1}\right\} \text {, }
\end{aligned}
$$

where

$$
\eta_{1}=\frac{1}{\Gamma(\alpha-p)}+\frac{1}{\Gamma(\alpha)}, \quad \eta_{2}=\frac{1}{\Gamma(\beta-q)}+\frac{1}{\Gamma(\beta)}
$$

The operator $K_{P}(I-Q) N: Y \rightarrow Y$ is continuous in view of the continuity of the function $f$ and $g$. Let $\Omega \subset Y$ be bounded; that is to say, there exists a positive constant $M>0$ such that $\|(x, y)\|_{Y} \leq M$ for all $(x, y) \in \Omega$.

$$
\text { We can derive that }
$$

$$
\begin{aligned}
& \max _{(x, y) \in \Omega}\left|K_{P}(I-Q) N(x, y)\right| \\
& =\max _{(x, y) \in \Omega}\left\{\left|I_{0+}^{\alpha}\{N x-Q N x\}\right|,\left|I_{0+}^{\beta}\{N y-Q N y\}\right|\right\} \\
& =\max _{(x, y) \in \Omega}\left\{\left|I_{0+}^{\alpha}\left\{f\left(t, x(t), D_{0+}^{p} x\right)-Q\left(f\left(t, x(t), D_{0+}^{p} x\right)\right)\right\}\right|,\right. \\
& \left.\quad\left|I_{0+}^{\beta}\left\{g\left(t, y(t), D_{0+}^{q} x\right)-Q\left(g\left(t, y(t), D_{0+}^{q} y\right)\right)\right\}\right|\right\}
\end{aligned}
$$




$$
\begin{gathered}
\leq \max _{(x, y) \in \Omega}\left\{\frac{1}{\Gamma(\alpha)} \| g\left(t, y(t), D_{0+}^{q} y\right)\right. \\
-Q\left(g\left(t, y(t), D_{0+}^{q} y\right)\right) \|_{1} \\
\frac{1}{\Gamma(\beta)} \| g\left(t, y(t), D_{0+}^{q} y\right) \\
\leq \max _{(x, y) \in \Omega}\left\{\frac{1}{\Gamma(\alpha)} M_{1}, \frac{1}{\Gamma(\beta)} M_{2}\right\} .
\end{gathered}
$$

Here,

$$
\begin{aligned}
& M_{1}=\max _{\|x\|_{C^{p}} \leq M}\left\|f\left(t, x(t), D_{0+}^{p} x\right)-Q\left(f\left(t, x(t), D_{0+}^{p} x\right)\right)\right\|_{1}, \\
& M_{2}=\max _{\|y\|_{C^{q}} \leq M}\left\|f\left(t, y(t), D_{0+}^{q} y\right)-Q\left(f\left(t, y(t), D_{0+}^{q} y\right)\right)\right\|_{1} .
\end{aligned}
$$

By Lemma 5,

$$
\begin{aligned}
& \max _{x \in \Omega}\left|D_{0+}^{p} K_{P}(I-Q) N x\right| \\
& \quad=\max _{x \in \Omega}\left|D_{0+}^{p} I_{0+}^{\alpha}\{N x-Q N x\}\right| \\
& \quad=\max _{x \in \Omega}\left|I_{0+}^{\alpha}\left\{f\left(t, x(t), D_{0+}^{p} x\right)-Q\left(f\left(t, x(t), D_{0+}^{p} x\right)\right)\right\}\right| \\
& \quad \leq \frac{1}{\Gamma(\alpha-p)}\left\|f\left(t, x(t), D_{0+}^{p} x\right)-Q\left(f\left(t, x(t), D_{0+}^{p} x\right)\right)\right\|_{1} \\
& \quad \leq \frac{1}{\Gamma(\alpha-p)} M_{1} .
\end{aligned}
$$

Similarly, we can prove that $\max _{y \in \Omega}\left|D_{0+}^{q} K_{P}(I-Q) N y\right| \leq$ $(1 / \Gamma(\beta-q)) M_{2}$.

Hence,

$$
\begin{aligned}
&\left\|K_{P}(I-Q) N(x, y)\right\|_{Y} \\
& \leq \max _{(x, y) \in \Omega}\left\{\left(\frac{1}{\Gamma(\alpha-p)}+\frac{1}{\Gamma(\alpha)}\right) M_{1},\right. \\
&\left.\left(\frac{1}{\Gamma(\beta-q)}+\frac{1}{\Gamma(\beta)}\right) M_{2}\right\},
\end{aligned}
$$

that is to say, $K_{P}(I-Q) N(\Omega) \subset Y$ is bounded. On the other hand, let

$$
\begin{aligned}
M_{3} & =\max _{\|x\|_{Y_{1}} \leq M} \max _{t \in J}\left|f\left(t, x(t), D_{0+}^{p} x\right)-Q\left(f\left(t, x(t), D_{0+}^{p} x\right)\right)\right| \\
& <+\infty
\end{aligned}
$$

$$
\begin{aligned}
M_{4} & =\max _{\|y\|_{Y_{2}} \leq M} \max _{t \in J}\left|f\left(t, y(t), D_{0+}^{q} y\right)-Q\left(f\left(t, y(t), D_{0+}^{q} y\right)\right)\right| \\
& <+\infty .
\end{aligned}
$$

For every $(x, y) \in \Omega, 0 \leq t_{1} \leq t_{2} \leq 1$, for all $\varepsilon>0$, there is $\delta=\min \left\{\left(\Gamma(\alpha-p) /\left(M_{1}+1\right)\right) \varepsilon,\left(\Gamma(\beta-q) /\left(M_{2}+1\right)\right) \varepsilon\right\}$, and $t_{2}-t_{1}<\delta$ such that

$$
\begin{gathered}
\left|D_{0+}^{p} K_{P}(I-Q) N x\left(t_{2}\right)-D_{0+}^{p} K_{P}(I-Q) N x\left(t_{1}\right)\right| \\
=\mid I_{0+}^{\alpha-p}\left[f\left(t_{2}, x\left(t_{2}\right), D_{0+}^{p} x\left(t_{2}\right)\right)\right. \\
\left.\quad-Q\left(f\left(t_{2}, x\left(t_{2}\right), D_{0+}^{p} x\left(t_{2}\right)\right)\right)\right] \\
\quad-I_{0+}^{\alpha-p}\left[f\left(t_{1}, x\left(t_{1}\right), D_{0+}^{p} x\left(t_{1}\right)\right)\right. \\
\left.\quad-Q\left(f\left(t_{1}, x\left(t_{1}\right), D_{0+}^{p} x\left(t_{1}\right)\right)\right)\right] \mid \\
=\frac{1}{\Gamma(\alpha-p)} \mid \int_{t_{1}}^{t_{2}}(t-s)^{\alpha-p-1} \\
\quad \times\left[f\left(s, x(s), D_{0+}^{p} x(s)\right)\right. \\
\left.\quad-Q\left(f\left(s, x(s), D_{0+}^{p} x(s)\right)\right)\right] d s \mid \\
\leq \frac{1}{\Gamma(\alpha-p)} M_{1} \delta<\varepsilon, \\
\left|D_{0+}^{q} K_{P}(I-Q) N y\left(t_{2}\right)-D_{0+}^{q} K_{P}(I-Q) N y\left(t_{1}\right)\right|<\varepsilon
\end{gathered}
$$

This ensures that $K_{P}(I-Q) N(\Omega)$ is equicontinuous too. Then an application of Ascoli-Arezela theorem ensures that $K_{P}(I-$ Q) $N: Y \rightarrow Y$ is completely continuous.

Theorem 14. Assume that $f, g:[0,1] \times R^{2} \rightarrow R$ satisfy the Carathéodory conditions, $t \in[0,1], \Lambda_{1}, \Lambda_{2} \neq 0,1<\alpha, \beta \leq$ $2,0<p, q<1, \alpha-p-1 \geq 0, \beta-q-1 \geq 0$, and $\sum_{i=1}^{m-2} a_{i} \xi_{i}^{\alpha-p-1}=1, \sum_{i=1}^{m-2} b_{i} \eta_{i}^{\beta-q-1}=1$. Assume that all of the following hypotheses hold.

$\left(H_{1}\right)$ There exist nonnegative functions $a_{i}, b_{i}, c_{i} \in Z, i=$ $1,2, t \in J$, such that for all $(x, y) \in R^{2}$,

$$
\begin{aligned}
& |f(t, x, y)| \leq a_{1}(t)|x|+b_{1}(t)|y|+c_{1}(t), \\
& |g(t, x, y)| \leq a_{2}(t)|x|+b_{2}(t)|y|+c_{2}(t) .
\end{aligned}
$$

$\left(H_{2}\right) 0<\eta_{1}^{\prime} \eta_{1}^{\prime \prime}<1,0<\eta_{2}^{\prime} \eta_{2}^{\prime \prime}<1$, where $\eta_{1}, \eta_{2}$ is defined by (35) and $\eta_{1}^{\prime \prime}=\left\|a_{1}\right\|_{1}+\left\|b_{1}\right\|_{1}, \eta_{2}^{\prime \prime}=\left\|a_{2}\right\|_{1}+\left\|b_{2}\right\|_{1}$, $\eta_{1}^{\prime}=(\Gamma(p+1)+1) / \Gamma(\alpha-p)+\eta_{1}, \eta_{2}^{\prime}=(\Gamma(q+1)+$ $1) / \Gamma(\beta-q)+\eta_{2}$.

$\left(H_{3}\right)$ There exist constants $T_{1}, T_{2}>0$ such that $Q_{1}(N u) \neq 0$, $Q_{2}(N v) \neq 0$, for each $u \in \operatorname{dom} L_{1}, v \in \operatorname{dom} L_{2}$ satisfying $\left|D_{0+}^{p} u(t)\right|>T_{1},\left|D_{0+}^{q} v(t)\right|>T_{2}$, for all $t \in[0,1]$. 
$\left(H_{4}\right)$ There exist constants $S_{1}, S_{2}>0$ such that for any $c_{1}, c_{2} \in$ $R$, if $\left|c_{1}\right|>S_{1},\left|c_{2}\right|>S_{2}$, either

$$
c_{1} \Lambda_{1} Q_{1}\left(N\left(c_{1} t^{\alpha-1}\right)\right)<0, \quad c_{2} \Lambda_{2} Q_{2}\left(N\left(c_{2} t^{\beta-1}\right)\right)<0 \text {, }
$$

or else

$$
c_{1} \Lambda_{1} Q_{1}\left(N\left(c_{1} t^{\alpha-1}\right)\right)>0, \quad c_{2} \Lambda_{2} Q_{2}\left(N\left(c_{2} t^{\beta-1}\right)\right)>0 .
$$

Then the problem (3) has at least one solution in $\operatorname{dom} L$.

Proof. The proof consists of four main steps.

Step 1. Set $\Omega_{1}=\{(u, v) \in \operatorname{dom} L \backslash \operatorname{Ker} L \mid L(u, v)=$ $\lambda N(u, v), \lambda \in[0,1]\}$, and prove $\Omega_{1}$ is bounded. In fact, for $(u, v) \in \Omega_{1}$, then $(u, v) \in \operatorname{dom} L \backslash \operatorname{Ker} L$ and $L(u, v)=$ $\lambda N(u, v)$, so $\lambda \neq 0$ and $N(u, v) \in \operatorname{Im} L=\operatorname{Ker} Q \subset Z$. Hence, $Q(N(u, v))=0$, that is, $Q_{1}\left(N_{1} u(t)\right)=0, Q_{2}\left(N_{2} v(t)\right)=0$. From $\left(H_{3}\right)$, we have that $t_{0} \in[0,1]$ such that $\left|D_{0+}^{p} u\left(t_{0}\right)\right| \leq T_{1}$, $\left|D_{0+}^{q} v\left(t_{0}\right)\right| \leq T_{2}$.

Again, for $(u, v) \in \Omega_{1}$, we get

$$
\begin{aligned}
\|P u\|_{Y_{1}} & =\max _{u \in \Omega_{1}}\left|D_{0+}^{p} P u\right|+\max _{u \in \Omega_{1}}|P u| \\
& =\max _{u \in \Omega_{1}}\left|D_{0+}^{p}\left(\left(D_{0+}^{p} u\left(t_{0}\right)\right) t^{p}\right)\right|+\max _{u \in \Omega_{1}}\left|\left(D_{0+}^{p} u\left(t_{0}\right)\right) t^{p}\right| \\
& \leq\left|D_{0+}^{p} u\left(t_{0}\right)\right| \Gamma(p+1)+\left|D_{0+}^{p} u\left(t_{0}\right)\right|, \\
& \|P v\|_{Y_{2}} \leq\left|D_{0+}^{q} v\left(t_{0}\right)\right| \Gamma(q+1)+\left|D_{0+}^{q} v\left(t_{0}\right)\right| .
\end{aligned}
$$

In view of Lemma 5, we have

$$
\begin{aligned}
\left|D_{0+}^{p} u\left(t_{0}\right)\right| & =\left|D_{0+}^{p} u\left(t_{0}\right)-I_{0+}^{\alpha-p}\left(D_{0+}^{p} u\left(t_{0}\right)\right)\right| \\
& \leq\left|D_{0+}^{p} u\left(t_{0}\right)\right|+\frac{1}{\Gamma(\alpha-p)}\left\|D_{0+}^{p} u(t)\right\|_{1} \\
& \leq T_{1}+\frac{1}{\Gamma(\alpha-p)}\left\|L_{1} u\right\|_{1} \\
& \leq T_{1}+\frac{1}{\Gamma(\alpha-p)}\|N u\|_{1} .
\end{aligned}
$$

Similarly, we can also obtain $\left|D_{0+}^{q} v\left(t_{0}\right)\right| \leq T_{2}+(1 / \Gamma(\beta-$ q)) $\left\|L_{2} v\right\|_{1}$.

Thus,

$\|P(u, v)\|_{Y}$

$$
\begin{array}{r}
\leq \max \left\{[\Gamma(p+1)+1]\left[T_{1}+\frac{1}{\Gamma(\alpha-p)}\|N u\|_{1}\right],\right. \\
\left.[\Gamma(q+1)+1]\left[T_{2}+\frac{1}{\Gamma(\beta-q)}\|N v\|_{1}\right]\right\} .
\end{array}
$$

According to Lemma 13, we have

$$
\begin{aligned}
\|(I-P)(u, v)\|_{Y} & =\left\|K_{P} L(I-P)(u, v)\right\|_{Y} \\
& \leq \max \left\{\eta_{1}\|L(I-P) u\|_{1}, \eta_{2}\|L(I-P) v\|_{1}\right\} \\
& =\max \left\{\eta_{1}\|L u\|_{1}, \eta_{2}\|L v\|_{1}\right\} \\
& \leq \max \left\{\eta_{1}\|N u\|_{1}, \eta_{2}\|N v\|_{1}\right\} .
\end{aligned}
$$

Combining (48) and (49), we can obtain

$$
\begin{aligned}
\|(u, v)\|_{Y}= & \|(I-P) u+P u\|_{Y} \\
\leq & \|P(u, v)\|_{Y}+\|(I-P)(u, v)\|_{Y} \\
= & \max \left\{[\Gamma(p+1)+1] T_{1}+\eta_{1}\|N u\|_{1},\right. \\
& {\left.[\Gamma(q+1)+1] T_{2}+\eta_{2}\|N v\|_{1}\right\} . }
\end{aligned}
$$

From $\left(H_{1}\right)$, for each $u \in \Omega_{1}$, we have

$$
\begin{aligned}
\|N u\|_{1}= & \int_{0}^{1}|N u(s)| d s \\
= & \int_{0}^{1}\left|f\left(s, u(s), D_{0+}^{p} u(s)\right)\right| d s \\
\leq & \int_{0}^{1}\left|a_{1}(s)\right||u(s)| d s \\
& +\int_{0}^{1}\left|b_{1}(s)\right|\left|D_{0+}^{p} u(s)\right| d s+\int_{0}^{1}\left|c_{1}(s)\right| d s \\
\leq & \left(\int_{0}^{1}\left|a_{1}(s)\right| d s+\int_{0}^{1}\left|b_{1}(s)\right| d s\right)\|u\|_{Y_{1}}+\left\|c_{1}\right\|_{1} \\
= & \eta_{1}^{\prime \prime}\|u\|_{Y_{1}}+\eta_{3} .
\end{aligned}
$$

Also, we can obtain that

$$
\|N v\|_{1}=\int_{0}^{1}|N v(s)| d s \leq \eta_{2}^{\prime \prime}\|v\|_{Y_{2}}+\eta_{4},
$$

where $\eta_{3}=\left\|c_{1}\right\|_{1}, \eta_{4}=\left\|c_{2}\right\|_{1}$. Thus, by $\left(H_{2}\right)$ and (50)-(52) we can derive that

$$
\begin{array}{r}
\|(u, v)\|_{Y} \\
\leq \max \left\{\frac{[\Gamma(p+1)+1] T_{1}+\eta_{1} \eta_{3}}{1-\eta_{1} \eta_{1}^{\prime \prime}},\right. \\
\left.\frac{[\Gamma(q+1)+1] T_{2}+\eta_{2} \eta_{4}}{1-\eta_{2} \eta_{2}^{\prime \prime}}\right\},
\end{array}
$$

which clearly states that $\Omega_{1}$ is bounded.

Step 2. Set $\Omega_{2}=\{(u, v) \in \operatorname{Ker} L \mid N(u, v) \in \operatorname{Im} L\}$ and prove that $\Omega_{2}$ is bounded. Let $(u, v) \in \Omega_{2}$; then

$$
\begin{aligned}
(u, v) \in \operatorname{Ker} L=\{ & \left\{(u, v) \in \operatorname{dom} L \mid u=c_{1} t^{\alpha-1},\right. \\
& \left.v=c_{2} t^{\beta-1}, \forall c_{1}, c_{2} \in R, t \in(0,1)\right\} .
\end{aligned}
$$


Since $N(u, v) \in \operatorname{Im} L=\operatorname{Ker} Q$, we have $Q(N(u, v))=(0,0)$, that is, $Q_{1}\left(N\left(c_{1} t^{\alpha-1}\right)\right)=0, Q_{2}\left(N\left(c_{2} t^{\beta-1}\right)\right)=0$. Taking account of $\left(H_{4}\right),\left|c_{1}\right| \leq S_{1},\left|c_{2}\right| \leq S_{2}$, which implies that $\Omega_{2}$ is bounded.

Step 3. Set $\Omega_{3}=\left\{(u, v) \in \operatorname{Ker} L \mid-\lambda(u, v)+(1-\lambda) J_{N L}\right.$ $\mathrm{QN}(u, v)=(0,0), \lambda \in[0,1]\}$; if (35) holds, we will set

$$
\begin{gathered}
\Omega_{3}=\left\{(u, v) \in \operatorname{Ker} L \mid \lambda(u, v)+(1-\lambda) J_{N L} Q N(u, v)\right. \\
=(0,0), \lambda \in[0,1]\}
\end{gathered}
$$

if (44) holds, where $J_{N L}: \operatorname{Im} Q \rightarrow \operatorname{Ker} L$ is a linear isomorphism defined as

$$
\begin{array}{r}
J_{N L}\left(c_{1} t^{k}\right)=c_{1} t^{\alpha-1}, \quad J_{N L}\left(c_{2} t^{k}\right)=c_{2} t^{\beta-1}, \\
\forall c_{1}, c_{2} \in R, t \in(0,1) .
\end{array}
$$

And then we will prove that $\Omega_{3}$ is bounded. Without loss of generality, in the following part of the proof, we assume that (35) holds in $\left(H_{4}\right)$, for $(u, v) \in \Omega_{3}$; we have $u=c_{1} t^{\alpha-1}, v=$ $c_{2} t^{\beta-1}, c_{1}, c_{2} \in R, t \in[0,1]$, and $\lambda(u, v)=(1-\lambda) J_{N L} Q N(u, v)$ Thus,

$$
\begin{aligned}
& \lambda t^{\alpha-1}=(1-\lambda) \frac{Q_{1}\left(N\left(c_{1} t^{\alpha-1}\right)\right)}{c_{1} \Lambda_{1}} t^{\alpha-1}, \\
& \lambda t^{\beta-1}=(1-\lambda) \frac{Q_{2}\left(N\left(c_{2} t^{\beta-1}\right)\right)}{c_{2} \Lambda_{2}} t^{\beta-1} .
\end{aligned}
$$

Therefore, via $\left(H_{4}\right)$ and (35), we have $\left|c_{1}\right| \leq S_{1},\left|c_{2}\right| \leq S_{2}$, which show that $\Omega_{3}$ is bounded.

Step 4. Let $\Omega$ be an bounded open set such that $\Omega \supset$ $\bigcup_{i=1}^{3} \bar{\Omega}_{i}$ and prove that $\operatorname{deg}\left(\left.J_{N L} Q N\right|_{\operatorname{Ker} L}, \Omega \cap \operatorname{Ker} L, 0\right) \neq 0$. The operator $N$ is $L$-compact on $\bar{\Omega}$ due to the fact that $Q N(\bar{\Omega})$ is bounded and $K_{P, Q} N=K_{P}(I-P) N: \bar{\Omega} \rightarrow Y$ is compact by Lemma 13; then by Step 1 and Step 2, we have

(i) $L(u, v) \neq \lambda N(u, v)$ for each $((u, v), \lambda) \in[(\operatorname{dom} L \backslash$ $\operatorname{Ker} L) \cap \partial \Omega] \times(0,1)$;

(ii) $N(u, v) \notin \operatorname{Im} L$ for each $(u, v) \in \operatorname{Ker} L \cap \partial \Omega$.

Define $H((u, v), \lambda)=-\lambda I(u, v)+(1-\lambda) J_{N L} Q N(u, v)$, where $I$ is the identity operator in $Y$. According to the arguments in Step 3, we have $H((u, v), \lambda) \neq 0$, for all $(u, v) \in$ $\operatorname{Ker} L \cap \partial \Omega$, and therefore, via the homotopy property of degree, we obtain that

$$
\begin{aligned}
& \operatorname{deg}\left(\left.J_{N L} Q N\right|_{\text {Ker } L}, \Omega \cap \operatorname{Ker} L, 0\right) \\
& =\operatorname{deg}(H(\cdot, 0), \Omega \cap \operatorname{Ker} L, 0) \\
& =\operatorname{deg}(H(\cdot, 1), \Omega \cap \operatorname{Ker} L, 0) \\
& =\operatorname{deg}(-I, \Omega \cap \operatorname{Ker} L, 0)=-1 \neq 0,
\end{aligned}
$$

which certifies the condition (3) of Theorem 8. Then, applying Theorem 8, we conclude that the problem (3) has at least one solution in dom $L \cap \bar{\Omega}$. The proof is complete.

\section{Conclusion}

In this paper, we obtain the existence results of muti-point boundary value problem at resonance for a coupled system of nonlinear fractional differential equations by means of the coincidence degree theory.

\section{Conflict of Interests}

The authors declare that they have no competing interests.

\section{Authors' Contribution}

Ruijuan Liu completed the proof and wrote the initial draft. Chunhai Kou provided the problem and gave some suggestions of amendment. Ruijuan Liu then finalized the paper. All authors read and approved the final paper.

\section{Acknowledgment}

This work was supported by the Natural Science Foundation of China under Grant no. 11271248.

\section{References}

[1] D. Guo, V. Lakshmikantham, and X. Liu, Nonlinear Integral Equations in Abstract Spaces, vol. 373 of Mathematics and its Applications, Academic Publishers, Dordrecht, The Netherlands, 1996.

[2] A. Babakhani and V. Daftardar-Gejji, "Existence of positive solutions of nonlinear fractional differential equations," Journal of Mathematical Analysis and Applications, vol. 278, no. 2, pp. 434-442, 2003.

[3] D. Delbosco and L. Rodino, "Existence and uniqueness for a nonlinear fractional differential equation," Journal of Mathematical Analysis and Applications, vol. 204, no. 2, pp. 609-625, 1996.

[4] A. A. Kilbas, H. M. Srivastava, and J. J. Trujillo, Theory and Applications of Fractional Differential Equations, vol. 204 of North-Holland Mathematics Studies, Elsevier, Amsterdam, The Netherlands, 2006.

[5] A. A. Kilbas and J. J. Trujillo, "Differential equations of fractional order: methods, results and problems I," Applicable Analysis, vol. 78, no. 1-2, pp. 153-192, 2001.

[6] A. A. Kilbas and J. J. Trujillo, "Differential equations of fractional order: methods, results and problems II," Applicable Analysis, vol. 81, no. 2, pp. 435-493, 2002.

[7] N. Kosmatov, "Integral equations and initial value problems for nonlinear differential equations of fractional order," Nonlinear Analysis. Theory, Methods \& Applications, vol. 70, no. 7, pp. 25212529, 2009.

[8] V. Lakshmikantham, "Theory of fractional functional differential equations," Nonlinear Analysis. Theory, Methods \& Applications, vol. 69, no. 10, pp. 3337-3343, 2008.

[9] V. Lakshmikantham and A. S. Vatsala, "Basic theory of fractional differential equations," Nonlinear Analysis. Theory, Methods \& Applications, vol. 69, no. 8, pp. 2677-2682, 2008.

[10] M. Muslim, C. Conca, and A. K. Nandakumaran, "Approximation of solutions to fractional integral equation," Computers \& 
Mathematics with Applications, vol. 59, no. 3, pp. 1236-1244, 2010.

[11] K. S. Miller and B. Ross, An Introduction to the Fractional Calculus and Fractional Differential Equations, A Wiley-Interscience Publication, John Wiley \& Sons, New York, NY, USA, 1993.

[12] I. Podlubny, Fractional Differential Equations, vol. 198 of Mathematics in Science and Engineering, Academic Press, San Diego, Calif, USA, 1999, An introduction to fractional derivatives, fractional differential equations, to methods of their solution and some of their applications.

[13] M. Stojanović, "Existence-uniqueness result for a nonlinear $n$ term fractional equation," Journal of Mathematical Analysis and Applications, vol. 353, no. 1, pp. 244-255, 2009.

[14] M. ur Rehman and R. A. Khan, "Existence and uniqueness of solutions for multi-point boundary value problems for fractional differential equations," Applied Mathematics Letters, vol. 23, no. 9, pp. 1038-1044, 2010.

[15] Y. Zhang, Z. Bai, and T. Feng, "Existence results for a coupled system of nonlinear fractional three-point boundary value problems at resonance," Computers \& Mathematics with Applications, vol. 61, no. 4, pp. 1032-1047, 2011.

[16] X. Su, "Boundary value problem for a coupled system of nonlinear fractional differential equations," Applied Mathematics Letters, vol. 22, no. 1, pp. 64-69, 2009.

[17] V. Lakshmikantham and S. Leela, "A Krasnoselskii-Kreintype uniqueness result for fractional differential equations," Nonlinear Analysis. Theory, Methods \& Applications, vol. 71, no. 7-8, pp. 3421-3424, 2009.

[18] J. Mawhin, "Topological degree and boundary value problems for nonlinear differential equations," in Topological Methods for Ordinary Differential Equations, vol. 1537 of Lecture Notes in Mathematics, pp. 74-142, Springer, Berlin, Germany, 1993.

[19] Y. Zhang and Z. Bai, "Existence of solutions for nonlinear fractional three-point boundary value problems at resonance," Journal of Applied Mathematics and Computing, vol. 36, no. 1-2, pp. 417-440, 2011. 


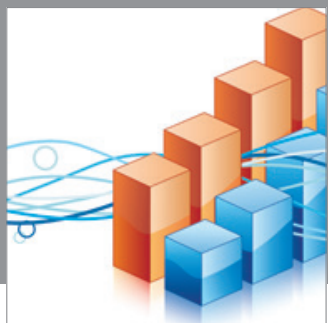

Advances in

Operations Research

mansans

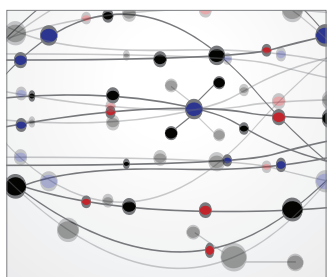

The Scientific World Journal
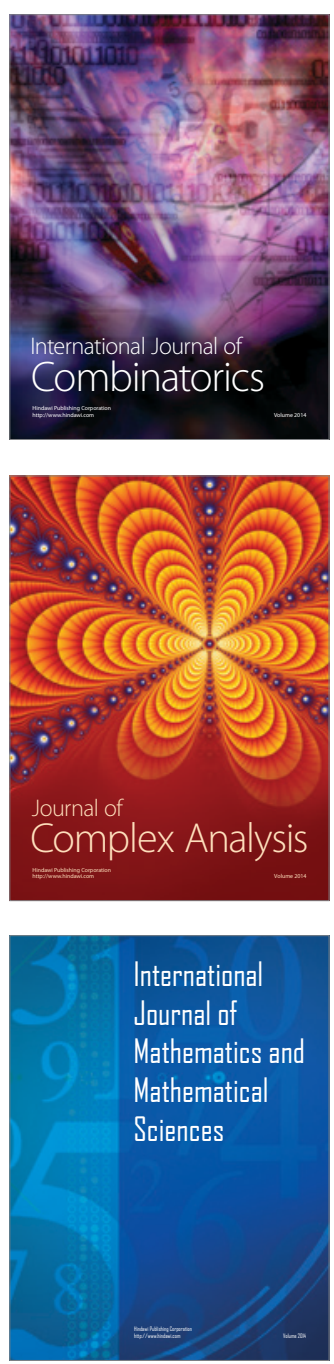
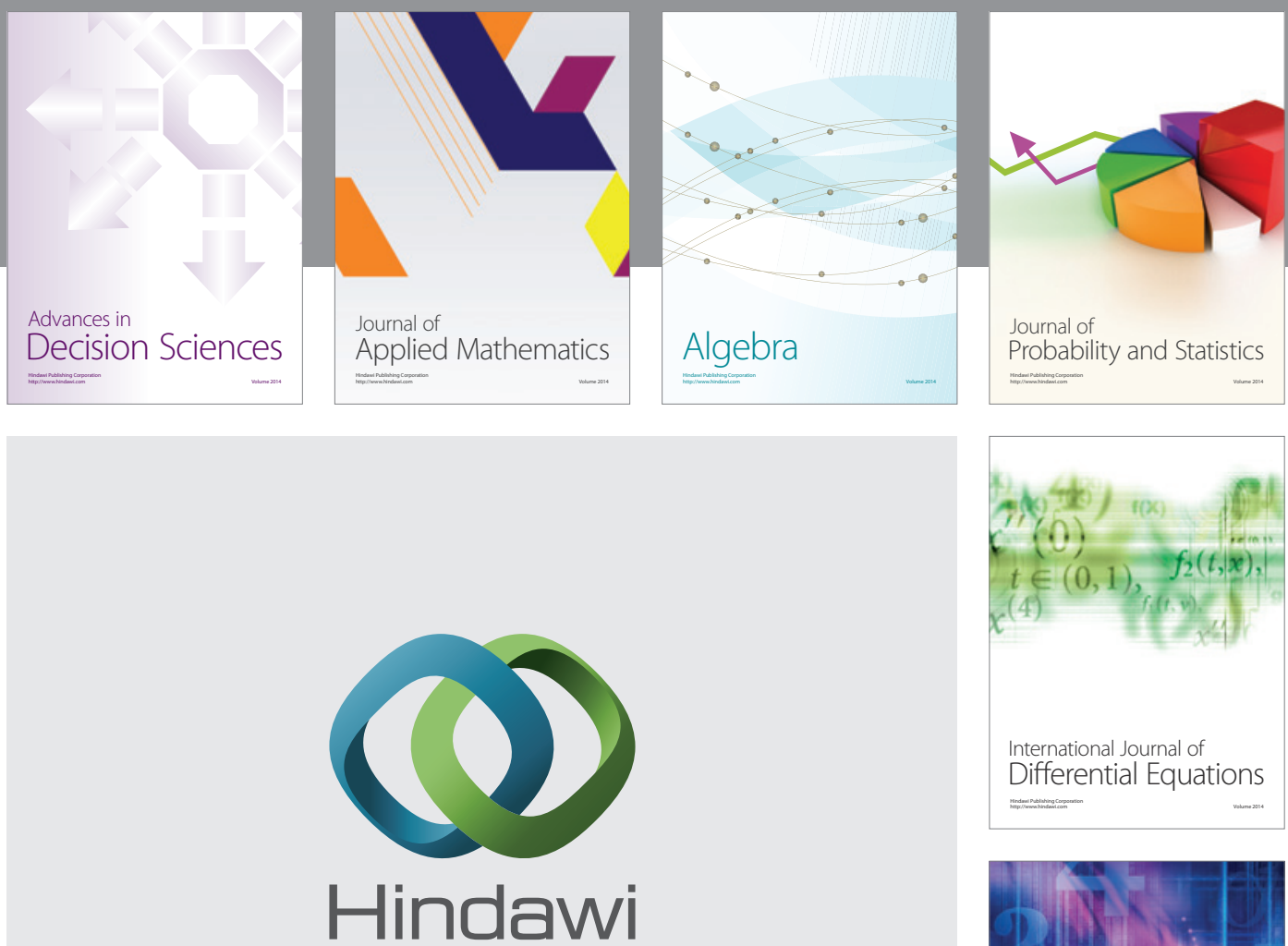

Submit your manuscripts at http://www.hindawi.com
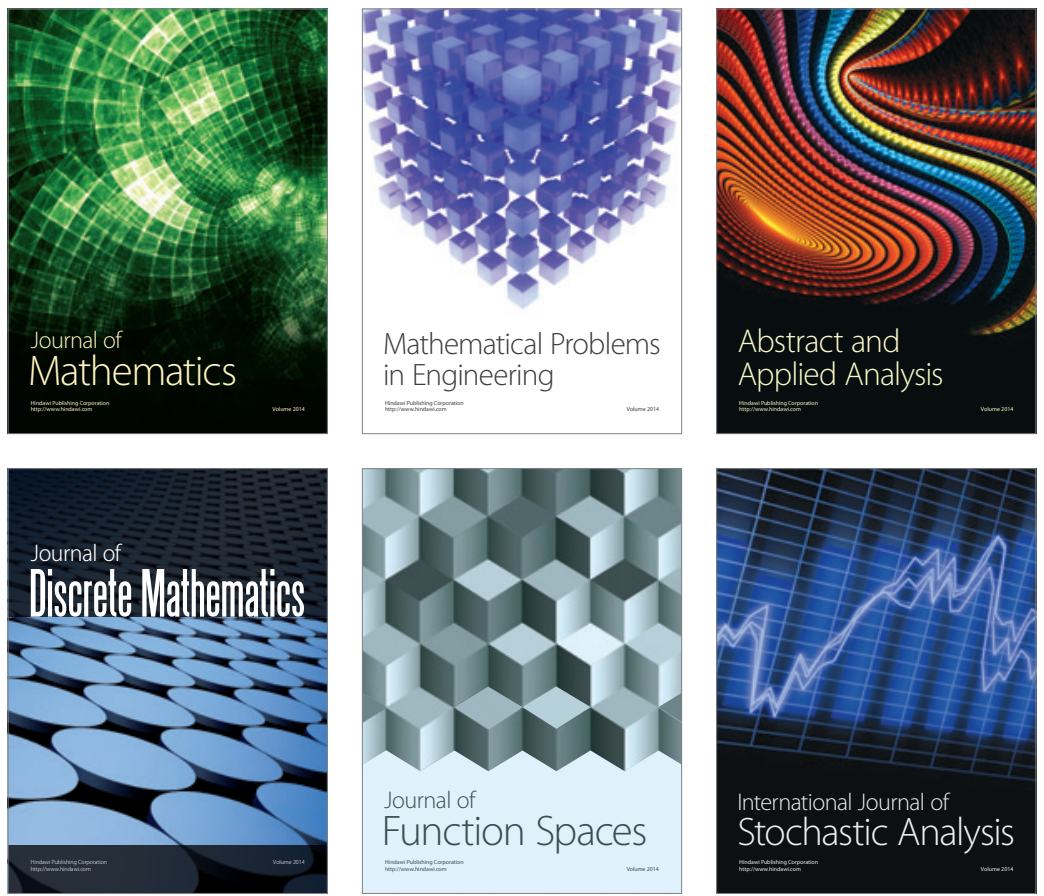

Journal of

Function Spaces

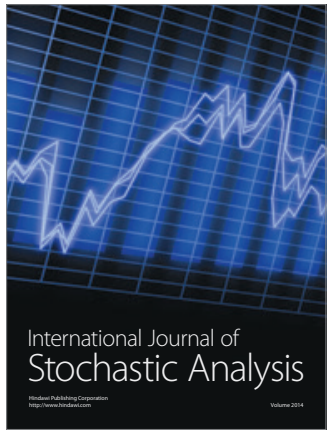

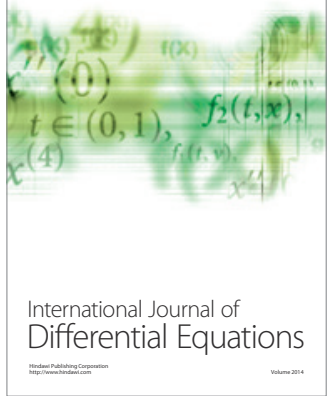
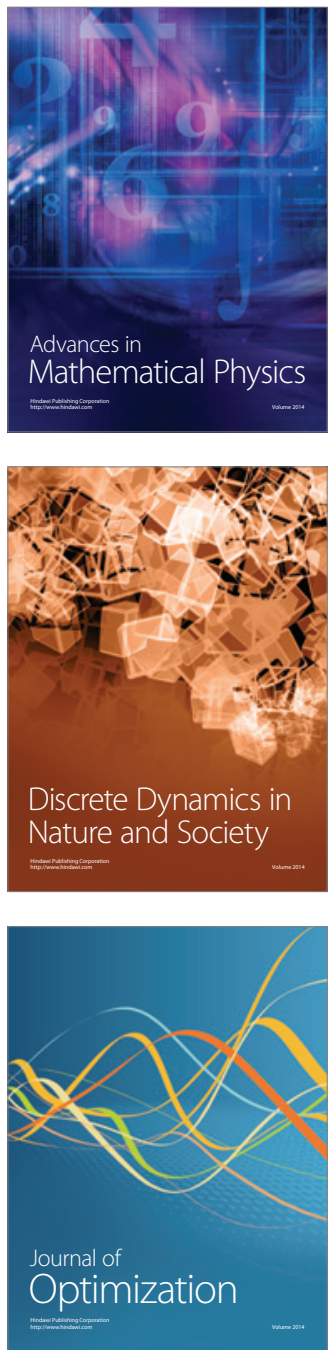